\title{
Thermo-chemotherapy inhibits the proliferation and metastasis of gastric cancer cells via suppression of EIF5A2 expression
}

This article was published in the following Dove Press journal: OncoTargets and Therapy

\author{
Ming-Chen $\mathrm{Ba}^{\mathrm{I}, *}$ \\ Zheng $\mathrm{Ba}^{2, *}$ \\ Shu-Zhong Cui' \\ Yuan-Feng Gong' \\ Cheng Chen' \\ Kun-Peng Lin' \\ Yin-Bing $\mathrm{Wu}^{\prime}$ \\ Yi-Nuo Tu'
}

'Intracelom Hyperthermic Perfusion Therapy Center, Affiliated Cancer Hospital \& Institute of Guangzhou Medical University, Guangzhou 510095 , People's Republic of China; ${ }^{2}$ Department of Intensive Care Unit, Zhujiang Hospital, Southern Medical University, Guangzhou 5 10282, People's Republic of China

*These authors contributed equally to this work

\begin{abstract}
Purpose: Thermo-chemotherapy (TCT) is a new approach for the treatment of cancer that combines chemotherapy with thermotherapy. In the present study, we investigated the relationship between eukaryotic translation initiation factor 5A2 (EIF5A2) and TCT sensitivity in gastric cancer (GC) to further illuminate the molecular mechanism underlying the effect of TCT on GC.

Methods: A TCT cell model was constructed, and EIF5A2 was silenced or overexpressed by infection with a lentivirus expressing either EIF5A2 or EIF5A2 shRNA. Then, RT-qPCR, Western blotting, and immunohistochemistry assays were performed to evaluate the changes in the expression levels of EIF5A2, c-myc, vimentin, and E-cadherin. Cell proliferation and xenograft assays were conducted to evaluate the effect on cell proliferation. Finally, wound-healing and Transwell invasion assays were performed to evaluate the effects on migration and invasion.

Results: TCT reduced EIF5A2 expression at both the mRNA and protein levels. It also inhibited cell proliferation, migration, and invasion, downregulated the expression of c-myc and vimentin, and increased the expression of E-cadherin in both MKN28 and MKN45 cells. Silencing of EIF5A2 enhanced the above effects of TCT on MKN28 and MKN45 cells, while overexpression of EIF5A2 had the opposite effects. In addition, EIF5A2 overexpression weakened the inhibitory effect of TCT on tumor growth in vivo as well as the effects on c-myc, vimentin, and E-cadherin. Conclusion: TCT inhibits GC cell proliferation and metastasis by suppressing EIF5A2 expression. Our results provide new insights into our understanding of the molecular mechanism underlying the effects of TCT in GC.
\end{abstract}

Keywords: gastric cancer, thermo-chemotherapy, EIF5A2, proliferation, epithelialmesenchymal transition

\section{Introduction}

Gastric cancer (GC) is a common malignancy and the second leading cause of cancer-related mortality worldwide. ${ }^{1}$ Surgery and chemotherapy are the major treatment approaches for improving the survival time of GC patients. However, the 5-year survival rate of patients with advanced GC remains unsatisfactory. ${ }^{2}$ Therefore, further elucidation of the molecular mechanism of GC progression and identification of novel, more effective clinical therapeutic strategies have become hotspots in GC research. Thermo-chemotherapy (TCT) is a new cancer treatment that combines chemotherapy with thermotherapy. Thermotherapy has synergistic effects when combined with chemotherapy, improves the outcomes of chemotherapy, and reduces its side effects, especially in advanced malignant tumors or tumors 
that are resistant to other treatments. ${ }^{3,4}$ Investigation of the molecular mechanism underlying the effects TCT in GC is imperative for improving the survival rate of patients with GC.

Eukaryotic translation initiation factor 5A2 (EIF5A2) is a member of the EIF5A gene family and is located on human chromosome 3q26.2. ${ }^{5}$ Numerous studies have demonstrated that EIF5A family genes function as novel oncogenes, and their expression is upregulated in various human cancers, such as pancreatic and prostate cancers. ${ }^{6-8}$ EIF5A2 plays an important role in tumor formation, cell proliferation, migration, invasion, and chemotherapeutic resistance. For example, silencing of EIF5A2 markedly suppressed cell proliferation, migration, and invasion and enhanced apoptosis and sensitivity to chemotherapeutics in non-small-cell lung cancer cells. ${ }^{9,10}$ Knockdown of EIF5A2 significantly inhibited the invasion and metastasis of hepatocellular carcinoma cells. ${ }^{11}$ Overexpression of EIF5A2 induced chemotherapy resistance to 5-fluorouracil in nasopharyngeal carcinoma cells. ${ }^{12}$ Inhibition of EIF5A2 activity by N1-guanyl-1,7-diaminoheptane enhanced the cisplatin chemosensitivity of oral squamous cell carcinoma cells. ${ }^{13}$ In addition, EIF5A2 promoted the progression of epithelial-mesenchymal transition (EMT) by inhibiting the expression of different downstream molecules in several cancers. ${ }^{14}$ Previous studies have demonstrated that the expression of EIF5A2 is related to cell aggressiveness and cisplatin resistance in GC cells as well as the poor survival of GC patients. ${ }^{15,16}$ However, the relationship between EIF5A2 expression and TCT sensitivity in GC cells remains largely unknown.

In this study, we investigated the effects of EIF5A2 expression on the proliferation, migration, invasion, and EMT of GC cells subjected to TCT to further elucidate the underlying molecular mechanism.

\section{Materials and methods}

\section{Cell culture and stable transfection}

The human GC cell lines MKN28 and MKN45 were purchased from the Cell Bank of Type Culture Collection of Chinese Academy of Sciences (Shanghai, China) and were cultured in RPMI 1640 medium (Gibco, Grand Island, NY, USA) supplemented with $10 \%$ fetal bovine serum (FBS; Gibco) at $37{ }^{\circ} \mathrm{C}$ in $5 \% \mathrm{CO}_{2}$ and $95 \%$ relative humidity. An shRNA construct targeting EIF5A2 clone in a lentivirus vector (pLVTHM-sh-EIF5A2, sh-EIF5A2), an EIF5A2 overexpression vector (PIRES2-EGFP-EIF5A2,
ov-EIF5A2), and respective negative control (NC) vectors, sh-NC and ov-NC, were purchased from Shanghai GenePharma (Shanghai, China). MKN28 and MKN45 cells displaying stable EIF5A2 overexpression or silencing were selected and cultured in appropriate antibiotic-containing RPMI 1640 medium. The expression of EIF5A2 was verified by RT-qPCR and Western blotting, and then the cells were used for subsequent experiments.

\section{Experimental design}

The experiment included six groups: 1) the cell group - cells cultured under "normal" conditions (in RPMI 1640 medium supplemented with $\mathrm{FBS}, 37{ }^{\circ} \mathrm{C}, 5 \% \mathrm{CO}_{2}$, and $95 \%$ relative humidity); 2) the TCT group - cells inoculated in medium containing $40 \mu \mathrm{g} / \mathrm{mL}$ oxaliplatin (Sigma-Aldrich, St. Louis, $\mathrm{MO}$, USA) and incubated at $43 \pm 0.2{ }^{\circ} \mathrm{C}\left(5 \% \mathrm{CO}_{2}\right.$ and $95 \%$ relative humidity) for $1 \mathrm{~h}$, and then cultured at $37{ }^{\circ} \mathrm{C}(5 \%$ $\mathrm{CO}_{2}$ and $95 \%$ relative humidity) for $23 \mathrm{~h}$, every day; 3 ) the TCT + sh-NC group - sh-NC cells treated as described for group 2 (TCT); 4) TCT + sh-EIF5A2 group - sh-EIF5A2 cells treated as described for group 2 (TCT); 5) TCT + ov-NC group - ov-NC cells treated as described for group 2 (TCT); and 6) TCT + ov-EIF5A2 group - ov-EIF5A2 cells treated as described for group 2 (TCT).

\section{RT-qPCR}

Total RNA was isolated from cells using TRIzol reagent (Invitrogen), according to the manufacturer's protocol. Total RNA $(1 \mu \mathrm{g})$ was then reverse transcribed to cDNA using the PrimeScript RT reagent kit (Takara, Dalian, China). mRNA expression was assessed using SYBR Green (Takara) and a 7500 real-time PCR system (Applied Biosystems, Foster City, CA, USA). The sequences of the primers used were as follows: EIF5A2 forward, 5'-GCTA CTGGTTGGCATTGTAA-3' and reverse, 5'-GGGCATGC TAATTGAGAGTT-3'-; E-cadherin forward, 5'-CCCACCA CGTACAAGGGTC-3' and reverse, 5'-CTGGGGTATTGG GGGCATC-3'; vimentin forward, 5'-CGCCAGATGCGT GAAATGG-3' and reverse, 5'-ACCAGAGGGAGTGAA TCCAGA-3'; c-myc forward, 5'-ACACATCAGCACAAC TACGC- $3^{\prime}$ and reverse, 5'-CCTCTTGACATTCTCCTCG GT-3'; and 18s rRNA forward, 5'-CCTGGATACCGCAGC TAGGA-3' and reverse, 5'-GCGGCGCAATACGAATGC CCC-3'. 18s rRNA was quantified as an endogenous control. Relative mRNA expression levels were calculated using the $2^{-\Delta \Delta \mathrm{Ct}}$ method. All experiments were performed in triplicate. 


\section{Western blotting}

After treatment, the GC cells were collected and lysed in icecold RIPA buffer (Beyotime, Shanghai, China) containing protease inhibitors according to the manufacturer's instructions. The protein concentration was determined using the BCA Protein Assay Kit (Beyotime). Equal amounts of protein were separated by sodium dodecyl-sulfate polyacrylamide gel electrophoresis and electrophoretically transferred to polyvinylidene fluoride membranes (Millipore, Billerica, MA, USA). The membranes were blocked with $5 \%$ non-fat dry milk in Tris-buffered saline (TBS) overnight at $4{ }^{\circ} \mathrm{C}$. The membranes were then incubated with the following primary antibodies at $25{ }^{\circ} \mathrm{C}$ for $2 \mathrm{~h}$ : anti-EIF5A2 antibody (1:1000; Abcam, Cambridge, MA, USA), anti-E-cadherin antibody (1:2000; Abcam, Cambridge, MA, UK), anti-vimentin antibody (1:2000; Abcam, Cambridge, MA, UK), anti-c-myc antibody (1:2000; Abcam, Cambridge, MA, UK), and antiGAPDH antibody (1:5000; Abcam, Cambridge, MA, UK). After washing with TBS containing Tween 20 (TBST) three times, the membrane was then incubated with a horseradish peroxidase-labeled secondary antibody $(1: 5000 ;$ Cell Signaling Technology, Danvers, MA, USA) at $25^{\circ} \mathrm{C}$ for $2 \mathrm{~h}$. The membranes were rinsed with TBST three times, and protein bands were visualized using the enhanced chemiluminescence kit (Thermo Scientific, Rockford, IL, USA). Relative protein expression was normalized to the expression of GAPDH.

\section{Cell proliferation assay}

The cell proliferation assay was performed using the Cell Counting Kit-8 (CCK-8; Beyotime), according to the manufacturer's protocol. GC cells $\left(4 \times 10^{3}\right.$ cells/well in $100 \mu \mathrm{L}$ of culture medium) were seeded in 96-well plates and allowed to attach for $12 \mathrm{~h}$. Then, the cells were treated as described in the Experimental design subsection and cultured for 0, 24, 48 , and $72 \mathrm{~h}$. Finally, CCK-8 reagent ( $10 \mu \mathrm{L} /$ well) was added to each well, mixed gently, and incubated at $37{ }^{\circ} \mathrm{C}$ for $2 \mathrm{~h}$. Then, the absorbance was read at $450 \mathrm{~nm}$ on a microplate reader. All assays were performed in triplicate.

\section{Wound healing experiment}

GC cells or transfected cells were seeded into six-well plates at a density of $2 \times 10^{5}$ cells/well and cultured for $24 \mathrm{~h}$ until the cells reached about $90 \%$ confluence. A scratch wound was made in the middle of the confluent monolayer using a sterile pipette tip, and the cells were washed with PBS three times. Then, medium containing $40 \mu \mathrm{g} / \mathrm{mL}$ oxaliplatin was added to the wells, and the cells were treated as described in the Experimental design subsection. At least four images of the scraped area were captured under a microscope (Olympus, Tokyo, Japan,) at $40 \times$ magnification at 0 and $24 \mathrm{~h}$. Closure of the wound was assessed, and wound closure is reported as a percentage compared to the size of the initial scratch. All assays were performed in triplicate.

\section{Transwell invasion assay}

A 24-well Transwell chamber (BD Biosciences, Franklin Lakes, NJ, USA) was used for the cell invasion assay. GC cells $\left(1 \times 10^{5}\right)$ were suspended in $300 \mu \mathrm{L}$ of serum-free medium and loaded into the upper chamber, which was pre-coated with Matrigel (BD Biosciences). The lower chamber was filled with $400 \mu \mathrm{L}$ of complete medium containing 10\% FBS. After treatment as described in the Experimental design subsection and culturing for $48 \mathrm{~h}$, the lower side of the filter was washed with PBS, fixed with methanol, and then stained with $0.5 \%$ crystal violet solution. The invading cells in each well were counted in three random high-power fields under a microscope (Olympus, Tokyo, Japan). Each experiment was performed in triplicate.

\section{Xenograft establishment and treatment}

All animal experiments were performed according to institutional Guidelines for Animal Care and Use and with the approval of the Animal Research Ethics Committee of Guangzhou Medical University. BALB/c athymic nude mice (male, 5 weeks old) were purchased from the Laboratory Animal Center of Guangzhou Medical University. The mice were randomly divided into four groups ( $n=4$ mice per group), which were maintained under pathogen-free conditions and treated as follows: mice in groups 1 and 2 were injected subcutaneously (in the left groin) with $5 \times 10^{6}$ either MKN28 (group 1) or MKN45 (group 2) cells (in $200 \mu \mathrm{L}$ ) that were infected with ov-NC; and mice in groups 3 and 4 were injected subcutaneously (in the left groin) with $5 \times 10^{6}$ either MKN28 (group 3) or MKN45 (group 4) cells (in $200 \mu \mathrm{L}$ ) infected with ov-EIF5A2. Tumor volumes were calculated using the following formula: volume $\left(\mathrm{mm}^{3}\right)=$ length $\times$ width $\times$ width $/ 2$. When the tumors reached a volume of approximately $300 \mathrm{~mm}^{3}$, the mice were injected intraperitoneally with oxaliplatin $(5 \mathrm{mg} / \mathrm{kg})$, and their left groin was immersed in a warm water tank at 43 $\pm 0.2{ }^{\circ} \mathrm{C}$ for $1 \mathrm{~h}$, and they were then fed under normal conditions, once every 3 days. Six days later, the animals were sacrificed, and tumor tissues were extracted for subsequent experiments. 


\section{Immunohistochemistry analysis}

Immunohistochemistry (IHC) analysis was performed on 4- $\mu \mathrm{m}$-thick sections of paraffin-embedded tumor tissue specimens. A monoclonal anti-human EIF5A2 antibody, anti-human E-cadherin antibody, anti-human vimentin antibody, and anti-human c-myc antibody were purchased from Cell Signaling Technology and were used for IHC according to the manufacturer's instructions. Briefly, 4- $\mu$ m-thick tissue sections cut from formalin-fixed, paraffin-embedded blocks were deparaffinized with xylene and rehydrated with ethanol. Endogenous peroxidase activity was blocked with 3\% $\mathrm{H}_{2} \mathrm{O}_{2}$ in methanol. After antigen retrieval, the sections were incubated with the primary antibodies overnight at $4{ }^{\circ} \mathrm{C}$. The sections were washed with PBS three times, and then incubated with a secondary antibody. After washing, a color reaction was induced by the IHC Streptavidin-Biotin Complex method and 3,3'-diaminobenzidine staining. The results of the IHC assay were independently reviewed by two senior pathologists who were blinded to the outcome of the study. The staining intensity of the target proteins was scored with reference to a previous study. ${ }^{17}$

\section{Statistical analysis}

Data were analyzed using SPSS 19.0 software (SPSS Inc., Chicago, IL, USA). The results are expressed as the mean \pm standard deviation (SD). Differences between two groups were analyzed using the Student's $t$-test. $P$-values less than 0.05 were considered statistically significant.

\section{Results}

\section{EIF5A2 expression levels in GC cells} subjected to TCT

The results showed that TCT reduced the mRNA and protein expression levels of EIF5A2 in both MKN28 and MKN45 cells. The mRNA and protein expression levels of EIF5A2 in the TCT + sh-EIF5A2 group were lower than those in the TCT + sh-NC group, while the mRNA and protein expression levels of EIF5A2 in the TCT + ov-EIF5A2 group were higher than those in the TCT + ov-NC group (Figure $1 \mathrm{~A}$ and $\mathrm{B}$ ).

\section{EIF5A2 expression and TCT influenced the proliferation of $\mathrm{GC}$ cells}

Cell proliferation assays showed that TCT inhibited the proliferation of both MKN28 and MKN45 cells, and silencing of EIF5A2 markedly inhibited the proliferation of both MKN28 and MKN45 cells subjected to TCT compared with the proliferation of control cells. Overexpression of EIF5A2 markedly promoted the proliferation of both MKN28 and MKN45 cells subjected to TCT compared with the proliferation of control cells (Figure 2). These results suggest that silencing of EIF5A2 enhanced the inhibitory effect of TCT on the proliferation of MKN28 and MKN45 cells, while overexpression of EIF5A2 had the opposite effect.

\section{EIF5A2 expression levels and TCT influenced the migration of GC cells}

A wound healing assay showed that TCT inhibited the migration of both MKN28 and MKN45 cells and silencing of EIF5A2 markedly inhibited the migration of both MKN28 and MKN45 cells subjected to TCT compared with the migration of control cells, while overexpression of EIF5A2 markedly promoted the migration of both MKN28 and MKN45 cells subjected to TCT compared with the migration of control cells (Figure 3). These results suggested that silencing of EIF5A2 enhanced the inhibitory effect of TCT on the migration of MKN28 and MKN45 cells, while overexpression of EIF5A2 had the opposite effect.

\section{EIF5A2 expression and TCT influenced the invasion of GC cells}

A Transwell cell invasion assay showed that TCT inhibited the invasion of both MKN28 and MKN45 cells and silencing of EIF5A2 markedly inhibited the invasion of both MKN28 and MKN45 cells subjected to TCT when compared with the invasion of control cells, while overexpression of EIF5A2 markedly promoted the invasion of both MKN28 and MKN45 cells subjected to TCT when compared with the invasion of control cells (Figure 4). These results suggested that silencing of EIF5A2 enhanced the inhibitory effect of TCT on the invasion of MKN28 and MKN45 cells, while overexpression of EIF5A2 had the opposite effect.

\section{EIF5A2 expression and TCT regulated the expression levels of c-myc, vimentin, and E-cadherin in GC cells}

The results showed that TCT reduced the mRNA and protein expression levels of c-myc and vimentin in both MKN28 and MKN45 cells, and silencing of EIF5A2 markedly reduced the mRNA and protein expression of c-myc and vimentin in 

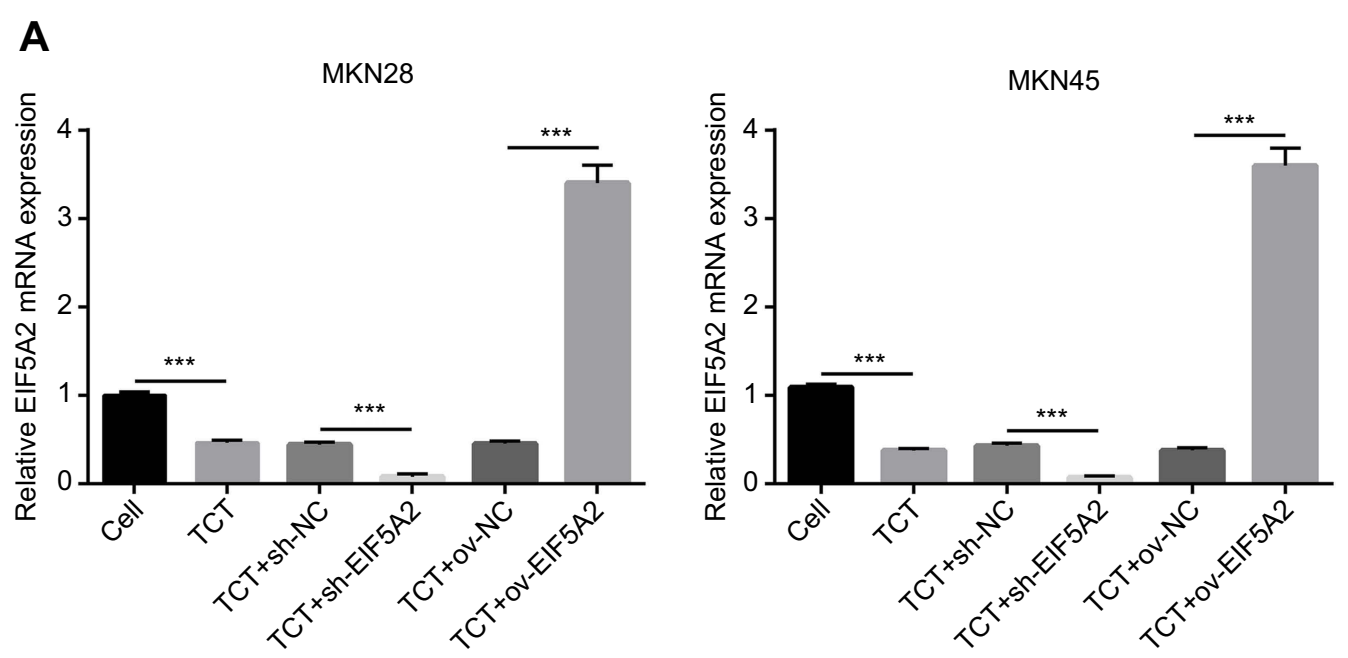

B

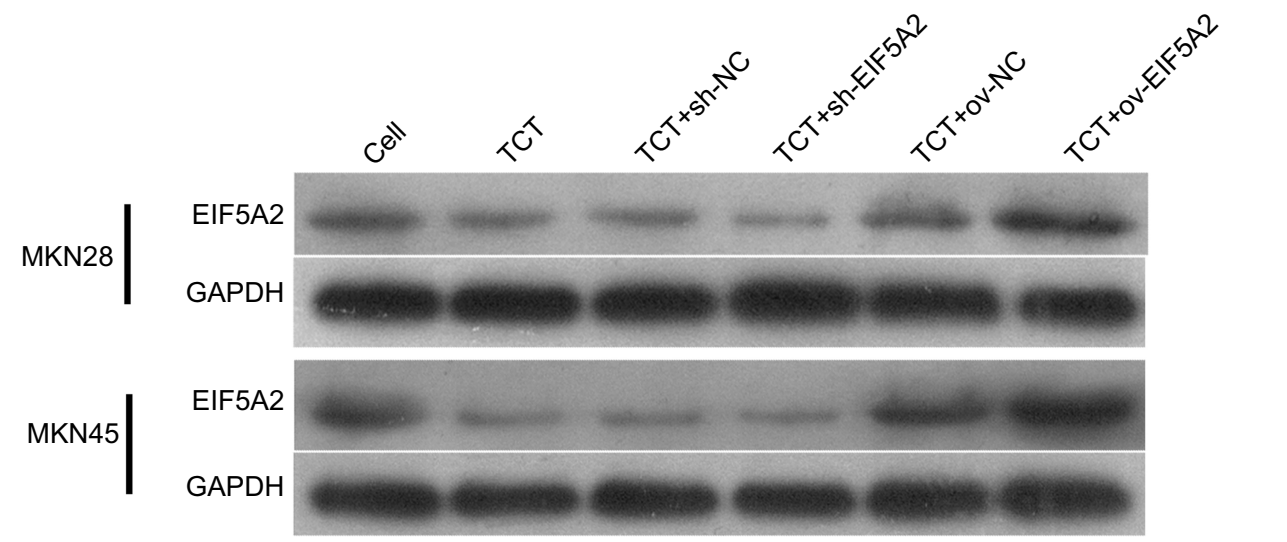

Figure I The expression levels of EIF5A2 in MKN28 and MKN45 cells subjected to thermo-chemotherapy (TCT). (A) The mRNA expression of EIF5A2 in MKN28 and MKN45 cells subjected to TCT was measured using RT-qPCR. (B) The protein expression of EIF5A2 in MKN28 and MKN45 cells subjected to TCT was analyzed using Western blotting. (***P<0.00I).

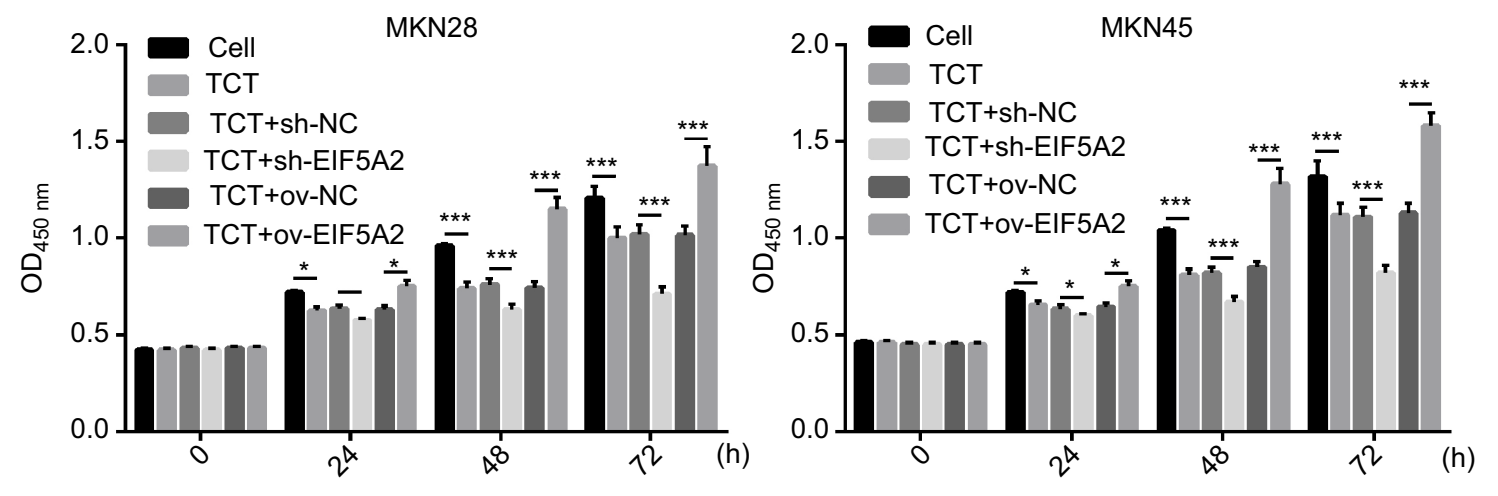

Figure 2 The effect of EIF5A2 expression and thermo-chemotherapy (TCT) on the proliferation of MKN28 and MKN45 cells was measured using the CCK-8 assay. *P<0.05, $* * * P<0.001$.

TCT treated MKN28 and MKN45 cells when compared with the levels in control cells, while overexpression of EIF5A2 had the opposite effect (Figure 5A, B, and D). TCT increased the mRNA and protein expression levels of E-cadherin in both MKN28 and MKN45 cells, and silencing of EIF5A2 markedly increased the mRNA and protein expression levels of E-cadherin in TCT-treated MKN28 and MKN45 cells when compared with the levels in control cells, while overexpression of EIF5A2 had the opposite effect (Figure 5C and D). 

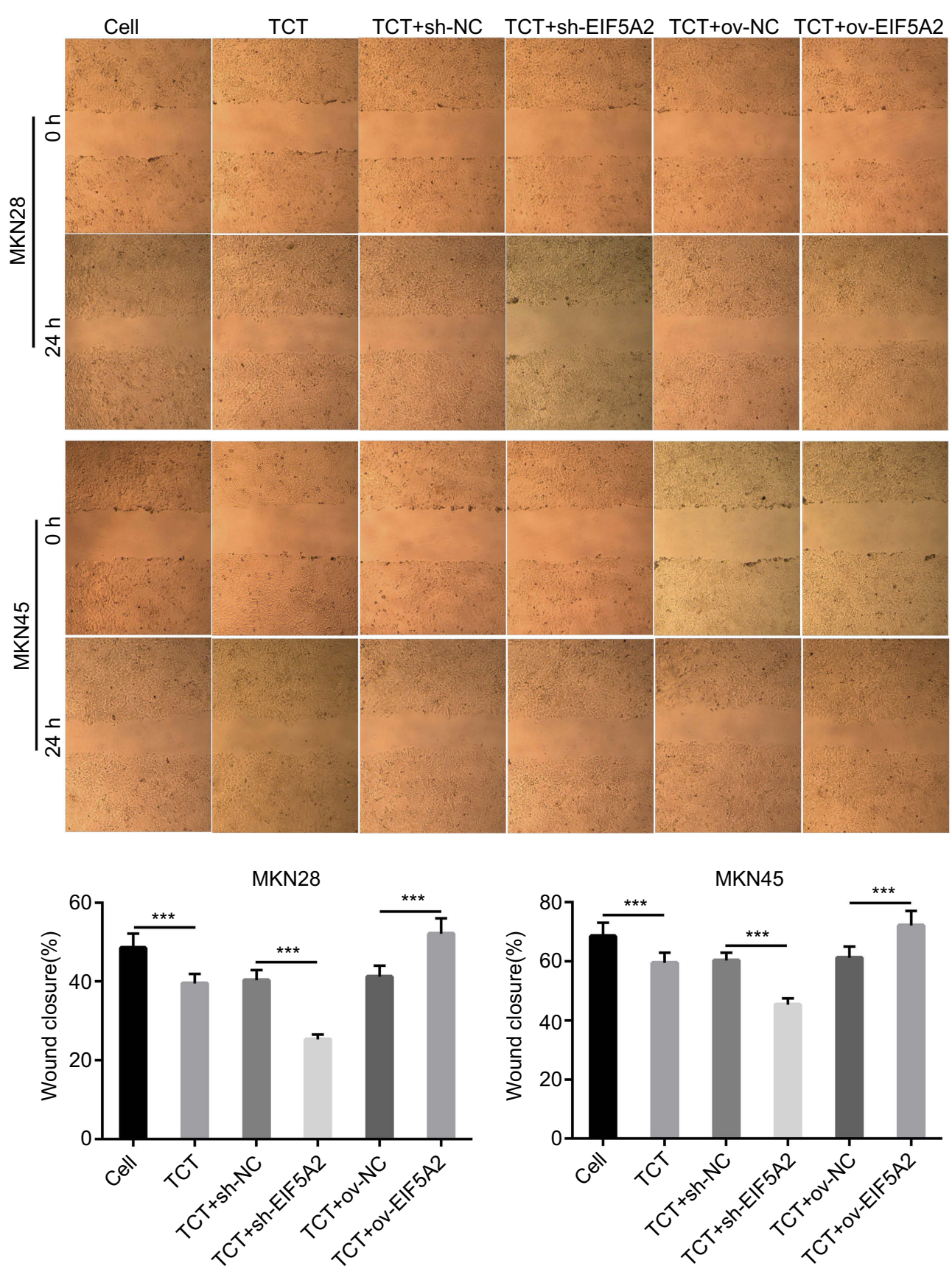

Figure 3 The effect of EIF5A2 expression and thermo-chemotherapy (TCT) on the migration of MKN28 and MKN45 cells was measured using a wound healing assay. The upper part is the representive images, and lower part is the statistical results of wound closure. $* * * P<0.001$.

\section{EIF5A2 overexpression weakened the} inhibitory effect of TCTon tumor growth in vivo

The effect of EIF5A2 overexpression and TCT on GC biology was further examined in vivo. The mean volume of ov-EIF5A2 MKN28 and MKN45 cellderived xenograft tumors subjected to TCT was significantly higher than that of ov-NC MKN28 and
MKN45 cell-derived xenograft tumors subjected to TCT (Figure 6A and B).

\section{EIF5A2 overexpression and TCT} regulated the expression levels of EIF5A2, c-myc, vimentin, and E-cadherin in vivo

Finally, the tumor sections were stained for EIF5A2, c-myc, vimentin, and E-cadherin expression. EIF5A2 

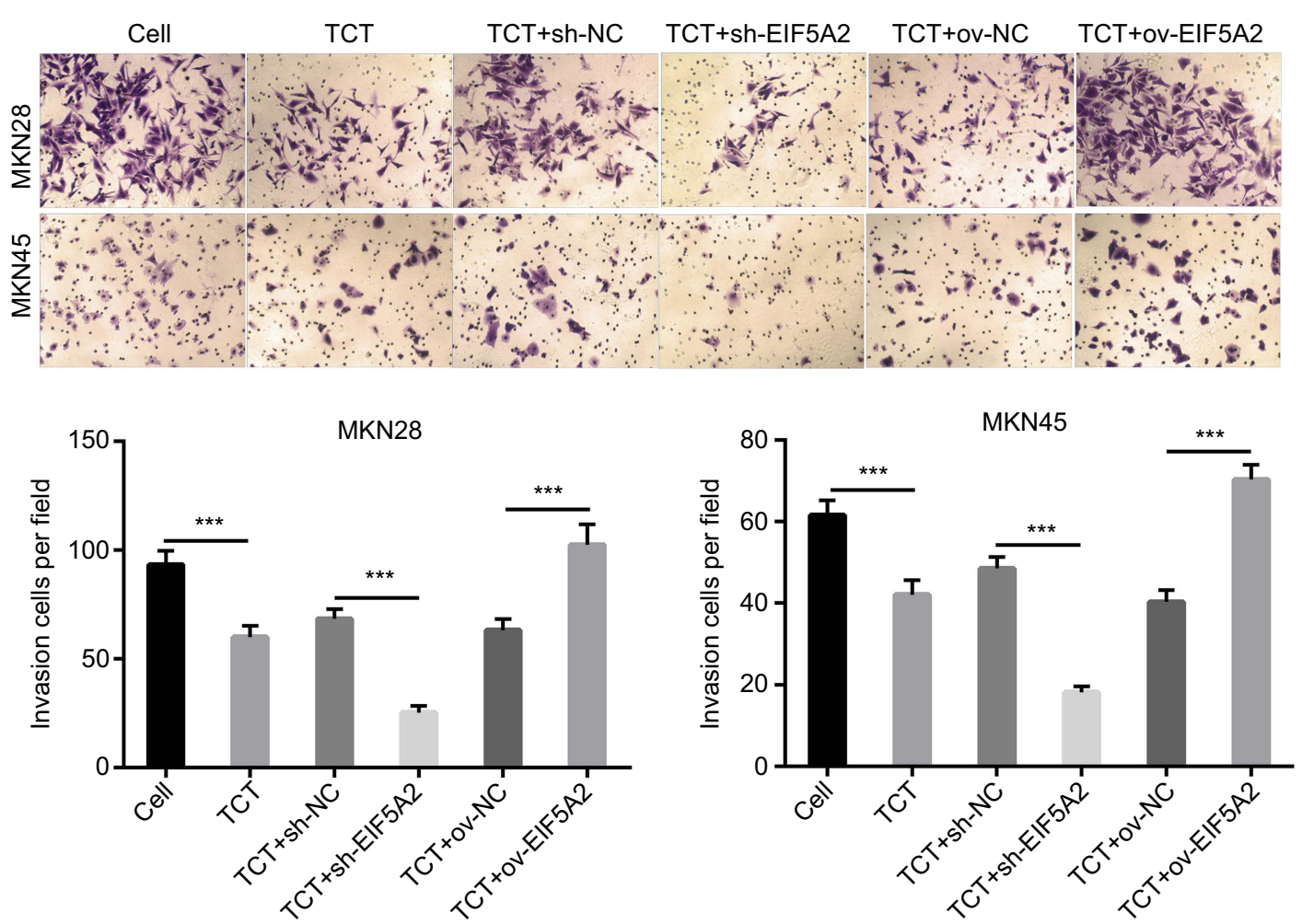

Figure 4 The effect of EIF5A2 expression and thermo-chemotherapy (TCT) on the proliferation of MKN28 and MKN45 cells was measured using a Transwell cell invasion assay. The upper part is the representive images, and the lower part is the statistical results of invasion cells per field. $* * * P<0.00 \mathrm{I}$.

A

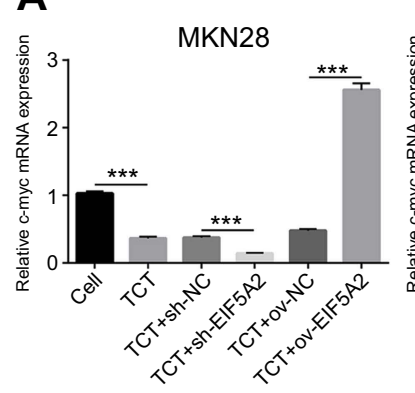

C

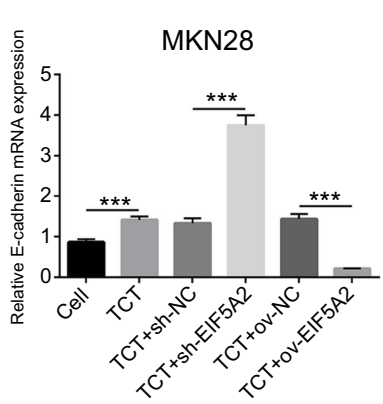

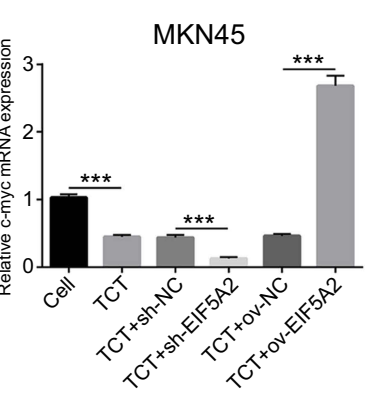

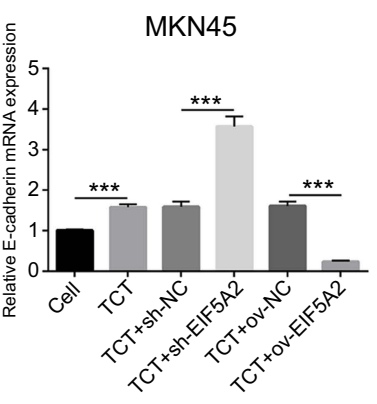

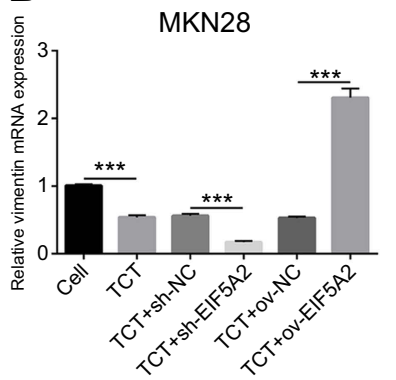

D

B
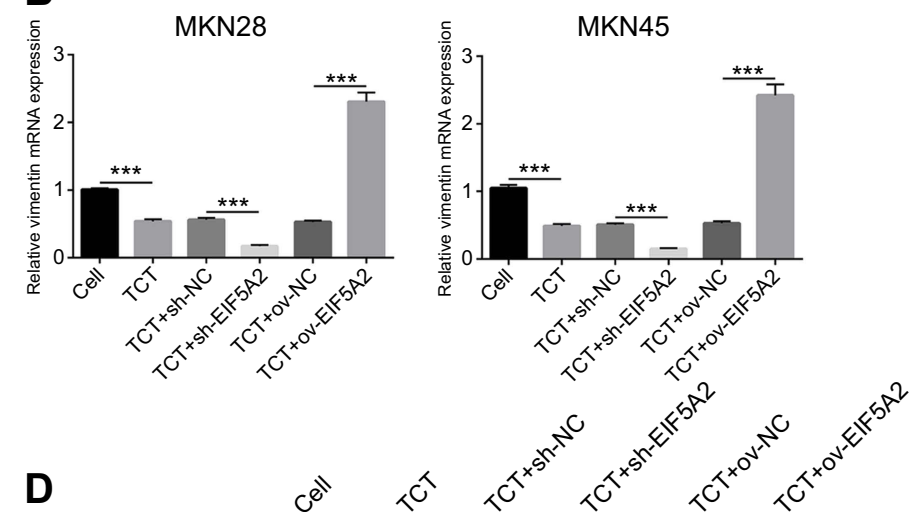
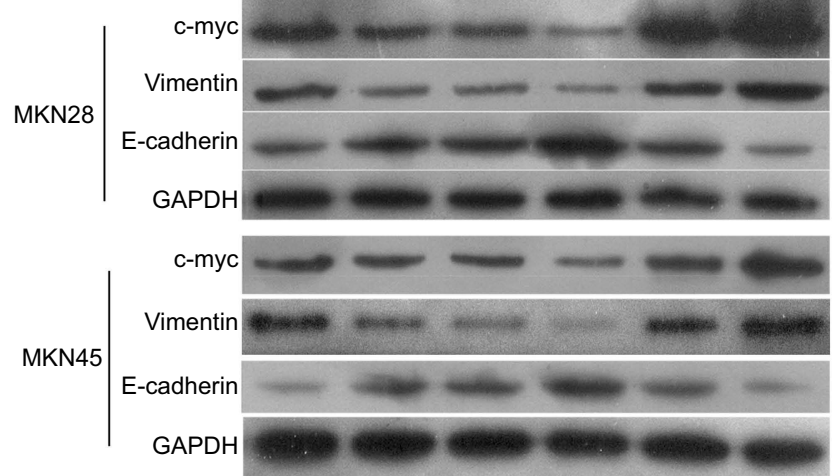

Figure 5 The effect of EIF5A2 expression and thermo-chemotherapy (TCT) on the mRNA and protein expression levels of c-myc, vimentin, and E-cadherin in MKN28 and MKN45 cells. (A-C) The mRNA expression levels of c-myc, vimentin, and E-cadherin in MKN28 and MKN45 cells subjected to TCT were measured using RT-qPCR. (D) The protein expression levels of c-myc, vimentin, and E-cadherin in MKN28 and MKN45 cells subjected to TCT were measured using Western blotting. ***P<0.00I. 
A

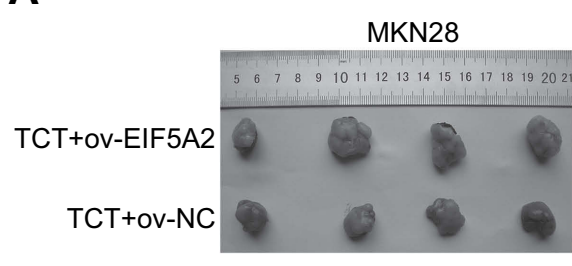

B

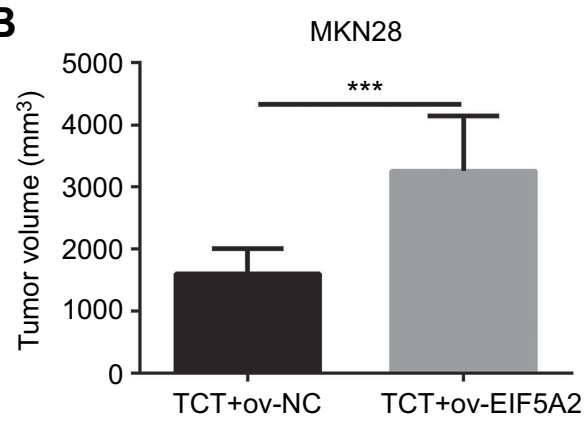

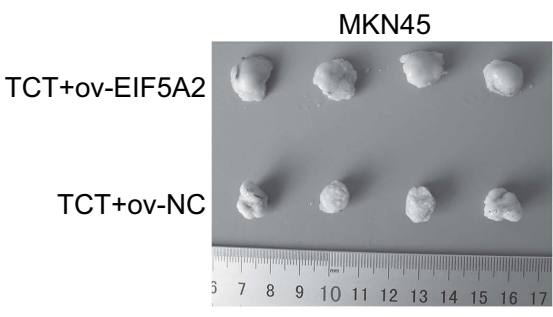

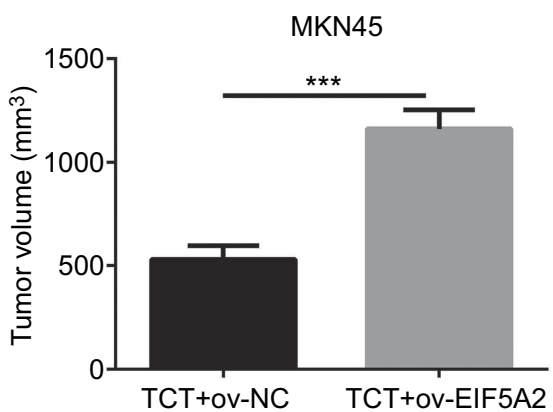

Figure 6 The effect of EIF5A2 overexpression and thermo-chemotherapy (TCT) on tumor growth in vivo. (A) Solid tumors were removed from mouse subcutaneous tissue. (B) The mean volume of ov-EIF5A2 or ov-NC cell-derived xenograft tumors generated from MKN28 and MKN45 cells subjected to TCT. ***P<0.00I.

was predominantly expressed in the nucleus of ovEIF5A2 cell-derived tumor cells. Compared with TCT-treated ov-NC cell-derived tumors cells, the expression levels of EIF5A2, c-myc, and vimentin in TCT-treated ov-EIF5A2 cell-derived tumors cells were significantly higher, while the expression of E-cadherin in TCT-treated ov-EIF5A2 cell-derived tumors cells was significantly lower (Figure 7).

\section{Discussion}

Several studies have reported that TCT is one of the most promising novel multidisciplinary approaches for cancer

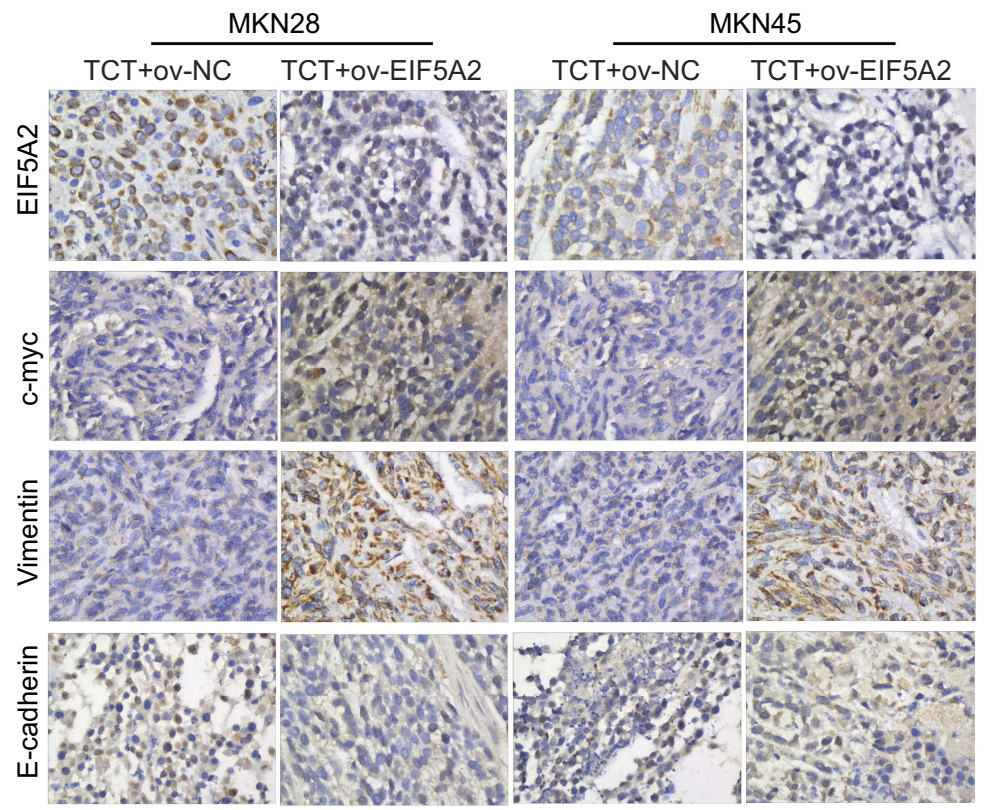

Figure 7 Representative immunohistochemical images showing the expression levels of EIF5A2, c-myc, vimentin, and E-cadherin in ov-EIF5A2 or ov-NC cell-derived tumor cells of MKN28 and MKN45 subjected to thermo-chemotherapy (TCT). 
treatment. $^{18}$ However, the mechanism underlying the effect of TCT in GC is still largely unknown. EIF5A2 has been identified as an oncogene and may be an important biomarker for prognosis and a useful therapeutic target in many types of human cancer. ${ }^{19}$ Numerous studies have confirmed that EIF5A2 plays an important role in the regulation of cell proliferation, apoptosis, and metastasis in many human cancers, such as ovarian and colorectal cancers. $^{20,21}$ Previous studies have shown that the expression of EIF5A2 was upregulated in GC tissues and cells; knockdown of EIF5A2 significantly inhibited cell proliferation, migration, and invasion and increased the sensitivity of GC cells to cisplatin; and overexpression of EIF5A2 was correlated with poorer survival in GC patients. ${ }^{15,16,22}$ In this study, we found that TCT reduced EIF5A2 expression; therefore, EIF5A2 may play a critical role in the inhibitory effect of TCT. Our present results provide new insight into the molecular mechanism underlying the effects of TCT in GC.

We found that TCT suppressed the expression of EIF5A2 and inhibited the proliferation, migration, and invasion of both MKN28 and MKN45 cells. Silencing of EIF5A2 markedly enhanced the inhibitory effects of TCT on the proliferation, migration, and invasion of both MKN28 and MKN45 cells, while overexpression of EIF5A2 had the opposite effects. Furthermore, animal experiments showed that EIF5A2 overexpression reversed the inhibitory effect of TCT on tumor growth. These findings suggested that EIF5A2 expression plays an important role in the mechanism of action of TCT in GC. This is the first study to report that EIF5A2 is involved in the in the inhibitory effects of TCT on cancer.

To further illuminate the underlying mechanism, we examined the expression levels of c-myc and two EMTassociated markers, E-cadherin and vimentin, in MKN28 and MKN45 cells subjected to TCT. The results showed that TCT reduced c-myc and vimentin expression levels but increased E-cadherin expression. Silencing of EIF5A2 significantly enhanced the effect of TCT on the expression levels of c-myc, vimentin, and E-cadherin, while overexpression of EIF5A2 had the opposite effect. Furthermore, animal tumor tissue IHC analysis showed that EIF5A2 overexpression increased the expression levels of c-myc and vimentin and decreased the expression of E-cadherin. c-Myc is a surface marker on cancer stem cells and an oncogene involved in tumorigenesis. ${ }^{23,24}$ Previous studies have reported that cmyc expression is correlated with chemotherapeutic resistance, ${ }^{25}$ and higher c-myc expression in GC patients led to worse prognosis. ${ }^{26}$ Therefore, we predicted that
EIF5A2 meditates the inhibitory effect of TCT on GC cell growth by regulating c-myc expression. EMT has been shown to be related to metastasis and invasiveness in various human tumors. ${ }^{27,28}$ Moreover, previous studies have demonstrated that EIF5A2 promotes cell invasion, migration, and EMT in various cancers. ${ }^{25,29}$ Therefore, we predicted that EIF5A2 mediates the inhibitory effect of TCT on GC cell metastasis by regulating the EMT. These results indicate that EIF5A2 overexpression increased the expression levels of cmyc and vimentin and decreased the expression level of Ecadherin, which weakened the inhibitory effect of TCT on GC cell proliferation and metastasis. Therefore, EIF5A2 may be a novel therapeutic target of TCT in GC.

In conclusion, the results of this study showed that TCT suppressed the proliferation and metastasis of GC cells by inhibiting EIF5A2 expression. Our study provided new insights into our understanding of the molecular mechanism underlying the effects of TCT in GC.

\section{Abbreviations}

EIF5A2, eukaryotic translation initiation factor 5A2; GC, gastric cancer; EMT, epithelial-mesenchymal transition; TCT, thermo-chemotherapy.

\section{Acknowledgments}

This work was supported by grants from the Guangzhou Key Medical Discipline Construction Project (No. 2017), the Guangdong Science and Technology Plan Project (No. 20160918), the Guangzhou Science Technology and Innovation Commission (No. 2014Y2-00152), and the Guangzhou Science Technology and Innovation Commission (No. 2014Y2-00548).

\section{Disclosure}

The authors have no conflicts of interest pertaining to this work.

\section{References}

1. Torre LA, Bray F, Siegel RL, Ferlay J, Lortet-Tieulent J, Jemal A Global cancer statistics, 2012. CA Cancer J Clin. 2015;65(2):87-108. doi: $10.3322 /$ caac. 21262

2. Thrumurthy SG, Chaudry MA, Chau I, Allum W. Does surgery have a role in managing incurable gastric cancer? Nat Rev Clin Oncol. 2015;12(11):676-682. doi:10.1038/nrclinonc.2015.132

3. Xiao F, Liu B, Zhu QX. c-Jun N-terminal kinase is required for thermotherapy-induced apoptosis in human gastric cancer cells. World $J$ Gastroenterol. 2012;18(48):7348-7356. doi:10.3748/wjg.v18.i48.7348

4. Kerbage Y, Betrouni N, Collinet P, et al. Laser interstitial thermotherapy application for breast surgery: current situation and new trends. Breast. 2017;33:145-152. doi:10.1016/j.breast.2017.03.016 
5. Jenkins ZA, Haag PG, Johansson HE. Human eIF5A2 on chromosome $3 \mathrm{q} 25-\mathrm{q} 27$ is a phylogenetically conserved vertebrate variant of eukaryotic translation initiation factor $5 \mathrm{~A}$ with tissue-specific expression. Genomics. 2001;71(1):101-109. doi:10.1006/geno.2000.6418

6. Mathews MB, Hershey JW. The translation factor eIF5A and human cancer. Biochim Biophys Acta. 2015;1849(7):836-844. doi:10.1016/j. bbagrm.2015.05.002

7. Lu J, Zhao HW, Chen Y, et al. Eukaryotic translation initiation factor $5 \mathrm{~A} 2$ is highly expressed in prostate cancer and predicts poor prognosis. Exp Ther Med. 2019;17(5):3741-3747. doi:10.3892/etm.2019.7331

8. Wang Z, Jiang J, Qin T, Xiao Y, Han L. EIF5A regulates proliferation and chemoresistance in pancreatic cancer through the sHH signalling pathway. J Cell Mol Med. 2019;23(4):2678-2688. doi:10.1111/jcmm.14167

9. Chen C, Zhang B, Wu S, Song Y, Li J. Knockdown of EIF5A2 inhibits the malignant potential of non-small cell lung cancer cells. Oncol Lett. 2018;15(4):4541-4549. doi:10.3892/ol.2018.7832

10. Xu G, Yu H, Shi X, et al. Cisplatin sensitivity is enhanced in nonsmall cell lung cancer cells by regulating epithelial-mesenchymal transition through inhibition of eukaryotic translation initiation factor 5A2. BMC Pulm Med. 2014;14:174. doi:10.1186/1471-2466-14-174

11. Liu RR, Lv YS, Tang YX, et al. Eukaryotic translation initiation factor $5 \mathrm{~A} 2$ regulates the migration and invasion of hepatocellular carcinoma cells via pathways involving reactive oxygen species. Oncotarget. 2016;7(17):24348-24360. doi:10.18632/oncotarget.8324

12. Huang PY, Zeng TT, Ban X, et al. Expression of EIF5A2 associates with poor survival of nasopharyngeal carcinoma patients treated with induction chemotherapy. BMC Cancer. 2016;16:669. doi:10.1186/ s12885-016-2714-2

13. Fang L, Gao L, Xie L, Xiao G. GC7 enhances cisplatin sensitivity via STAT3 signaling pathway inhibition and eIF5A2 inactivation in mesenchymal phenotype oral cancer cells. Oncol Rep. 2018;39 (3):1283-1291. doi:10.3892/or.2017.6161

14. Liu Y, Liu R, Fu P, et al. N1-guanyl-1,7-diaminoheptane sensitizes estrogen receptor negative breast cancer cells to doxorubicin by preventing epithelial-mesenchymal transition through inhibition of eukaryotic translation initiation factor 5A2 activation. Cell Physiol Biochem. 2015;36(6):2494-2503. doi:10.1159/000430209

15. Meng QB, Kang WM, Yu JC, et al. Overexpression of eukaryotic translation initiation factor 5A2 (EIF5A2) correlates with cell aggressiveness and poor survival in gastric cancer. PLoS One. 2015;10(3): e0119229. doi:10.1371/journal.pone.0119229

16. Sun J, Xu Z, Lv H, et al. eIF5A2 regulates the resistance of gastric cancer cells to cisplatin via induction of EMT. Am J Transl Res. 2018;10(12):4269-4279.

17. He LR, Zhao HY, Li BK, et al. Overexpression of eIF5A-2 is an adverse prognostic marker of survival in stage I non-small cell lung cancer patients. Int J Cancer. 2011;129(1):143-150. doi:10.1002/ijc.25669
18. Tang Y, McGoron AJ. Increasing the rate of heating: a potential therapeutic approach for achieving synergistic tumour killing in combined hyperthermia and chemotherapy. Int $J$ Hyperthermia. 2013;29(2):145-155. doi:10.3109/02656736.2012.760757

19. Wang FW, Guan XY, Xie D. Roles of eukaryotic initiation factor $5 \mathrm{~A} 2$ in human cancer. Int J Biol Sci. 2013;9(10):1013-1020. doi:10.7150/ ijbs.7191

20. Yang GF, Xie D, Liu JH, et al. Expression and amplification of eIF-5A2 in human epithelial ovarian tumors and overexpression of EIF-5A2 is a new independent predictor of outcome in patients with ovarian carcinoma. Gynecol Oncol. 2009;112(2):314-318. doi:10.1016/j.ygyno.2008.10.024

21. Xie D, Ma NF, Pan ZZ, et al. Overexpression of EIF-5A2 is associated with metastasis of human colorectal carcinoma. Hum Pathol. 2008;39(1):80-86. doi:10.1016/j.humpath.2007.05.011

22. Yang Q, Ye Z, Zhang Q, Zhao Z, Yuan H. Expression of eukaryotic translation initiation factor 5A-2 (eIF5A-2) associated with poor survival in gastric cancer. Tumour Biol. 2016;37(1):1189-1195. doi:10.1007/s13277-015-3894-0

23. Hidayat M, Mitsuishi Y, Takahashi F, et al. Role of FBXW7 in the quiescence of gefitinib-resistant lung cancer stem cells in EGFRmutant non-small cell lung cancer. Bosn J Basic Med Sci. 2019. doi:10.17305/bjbms.2019.4227

24. Ramanan M, Pilli VS, Aradhyam GK, Doble M. Transcriptional regulation of microsomal prostaglandin $\mathrm{E}$ synthase 1 by the protooncogene, c-myc, in the pathogenesis of inflammation and cancer. Biochem Biophys Res Commun. 2017;482(4):556-562. doi:10.1016/j. bbrc.2016.11.073

25. Wang X, Jin Y, Zhang H, Huang X, Zhang Y, Zhu J. MicroRNA-599 inhibits metastasis and epithelial-mesenchymal transition via targeting EIF5A2 in gastric cancer. Biomed Pharmacother. 2018;97:473480. doi:10.1016/j.biopha.2017.10.069

26. Calcagno DQ, Leal MF, Assumpcao PP, Smith MA, Burbano RR. MYC and gastric adenocarcinoma carcinogenesis. World $J$ Gastroenterol. 2008;14(39):5962-5968. doi:10.3748/wjg.14.5962

27. Yeung KT, Yang J. Epithelial-mesenchymal transition in tumor metastasis. Mol Oncol. 2017;11(1):28-39. doi:10.1002/1878-0261.12017

28. Jolly MK, Ware KE, Gilja S, Somarelli JA, Levine H. EMT and MET: necessary or permissive for metastasis? Mol Oncol. 2017;11 (7):755-769. doi:10.1002/1878-0261.12083

29. Zhu W, Cai MY, Tong ZT, et al. Overexpression of EIF5A2 promotes colorectal carcinoma cell aggressiveness by upregulating MTA1 through C-myc to induce epithelial-mesenchymaltransition. Gut. 2012;61(4):562-575. doi:10.1136/gutjnl-2011-300207
OncoTargets and Therapy

\section{Publish your work in this journal}

OncoTargets and Therapy is an international, peer-reviewed, open access journal focusing on the pathological basis of all cancers, potential targets for therapy and treatment protocols employed to improve the management of cancer patients. The journal also focuses on the impact of management programs and new therapeutic agents and protocols on patient perspectives such as quality of life, adherence and satisfaction. The manuscript management system is completely online and includes a very quick and fair peer-review system, which is all easy to use. Visit http://www.dovepress.com/ testimonials.php to read real quotes from published authors. 